\title{
Analysis and design on airport safety information management system
}

\author{
Lin Yan \\ School of Air Transportation, Shanghai University of Engineering Science, China
}

\begin{abstract}
Airport safety information management system is the foundation of implementing safety operation, risk control, safety performance monitor, and safety management decision for the airport. The paper puts forward the architecture of airport safety information management system based on $\mathrm{B} / \mathrm{S}$ model, focuses on safety information processing flow, designs the functional modules and proposes the supporting conditions for system operation. The system construction is helpful to perfecting the long effect mechanism driven by safety information, continually increasing airport safety management level and control proficiency.
\end{abstract}

\section{Introduction}

Safety is the eternal theme of civil aviation. Ensuring aviation safety is the focus of the world aviation industry. In the "13th Five-Year" period, China will construct 74 new airports. Till 2020, the total number of airport in China will be about 260 . The increase in the number of airports and the promotion of the scale will inevitably make the airport safety management face a huge challenge. High quality safety information is the basic guarantee of airport safety management. A good airport safety information management system is of great significance to the sustainable and safe development of the airport.

\section{System requirements analysis}

At present, existing aviation safety information management system research focuses on the whole civil aviation or an individual company. The related research on airport is very few. The existing airport safety information management systems have some disadvantages, such as insufficient function, poor operability, difficult to expand and so on. A good airport safety information management system should be able to: (1) accumulate a large number of basic original safety information; (2) make investigation and treatment timely on unsafe incidents; (3) find the useful information from the safety information to support the decision and improve safety management level of airport; (4) facilitate information retrieval of different authorized person and provide statistical reports, security reports and so on; (5) provide materials for relevant personnel to learn systematically and draw lessons from typical cases;
(6) establish information sharing and exchange between airport subsidiary companies, airports and resident units.

The paper focuses on the overall design of a good airport safety information management system. With the system, the safety information can be collected comprehensively, the information transmission process is smooth, the information can be fully utilized, and the airport safety management level can be improved.

\section{System's data sources}

Airport business can be divided into aviation and nonaviation business. The units providing service at the airport include airport authorities, customs, border inspection, entry-exit inspection and quarantine, the airlines, the ground services company, trading companies, cleaning companies, security companies and so on, therefore, the sources of airport safety information are very wide.

Besides collecting the safety information requested by Civil Aviation Administration of China (CCAR-396R3), the airport safety information management system should also collect the information that affects passenger safety and airport operation. The specific types of safety information are divided into: people's unsafe behaviours, unsafe conditions of devices, unsafe environment, unsafe production process, other potential risks and so on.

From the perspective of information processing, system's data sources are divided into: mandatory reporting, voluntary reporting, security report, complaint, danger or hidden danger report, safety performance monitor, operation deviation report, industry's accident and incident investigation etc. 


\section{System architecture and business process}

\subsection{System architecture}

Group Company is the management mode of most of airports in China. An airport group company may manage multiple airports and related business. Information communication between the subsidiaries is carried out through the network. Therefore, with the design principle of practicality, maintainability, security and friendly operability, airport safety information management system can be designed based on $\mathrm{B} / \mathrm{S}$ mode, using Oracle as database management system, under the SS2H framework of the JavaEE platform. Client terminal uses the browser to access server's web page or web interface. Server uses a multitier architecture, including a presentation tier, a business logic tier, and a data storage tier. The logical structure diagram of the system is shown in figure 1 .

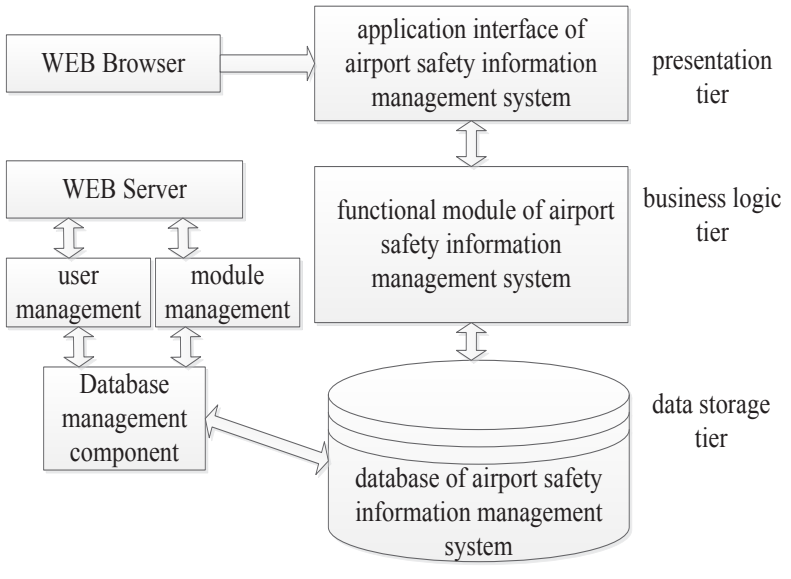

Fig. 1. Logical structure of the system

\subsection{System's business process}

The business process of airport safety information management system is divided into: information collection and collation, information analysis and evaluation, information release and sharing, information utilization, as shown in figure 2.

\subsubsection{Information collection and collation}

With the means of reports, records, networks, media, telephones, etc., People can submit various safety information of airport. Safety information personnel will categorize them into the system.

\subsubsection{Information analysis and evaluation}

Some safety information will trigger the incident investigation procedure, according to the results of the investigation, whether to further trigger the procedure of correction and prevention, risk control, or emergency rescue will be determined. The whole process of safety management activities will be recorded and monitored. At the same time, the airport safety situation is statistically analysed; the safety trends and laws are reflected, providing reference for leadership decisionmaking.

\subsubsection{Information release and sharing}

According to the content, nature and influence degree, the airport safety information which has been treated by certain procedures, can be published, spread and shared in different ways and scopes, such as safety meeting, internal propaganda, alarm information, etc. Typical cases can be chosen for safety education and training, to improve the safety awareness of employees and create a good safety culture atmosphere.

\subsubsection{Information utilization}

On the basis of information analysis and evaluation, information release and sharing, safety information can be further used by airport safety operation quality monitor, airport safety performance management, department safety assessment, airport regulation and standards revision, and so on.

\section{System's functional modules}

Based on the business process of the system, the functional modules of the system include safety information reporting module, safety supervision and audit module, incident investigation module, correction and prevention module, risk control module, emergency rescue module, data analysis module, safety notice module, safety education and training module, system management. They correspond to the input, processing and output of information management. The structure of functional modules is shown in Figure 3.

\subsection{Safety information reporting}

Safety information reporting module collects safety information from mandatory reporting, voluntary reporting, security report or complaint, danger or hidden danger report, industry's accident and incident investigation. The module provides special forms of information, and also provides a general form. Airport staffs or resident units can input information through the network. The information entered by the general form must be checked and classified by safety information personnel. Safety information can also be submitted by telephone, fax, mail, etc., then input by safety information personnel. The module can also query, modify and delete the information.

\subsection{Safety supervision and audit}

According to the airport safety situation and goal, safety supervision and audit module help to establish the plan 
of safety supervision and audit, completely record information of safety performance monitor and technical operation deviation, and automatically generate the safety supervision and audit report.

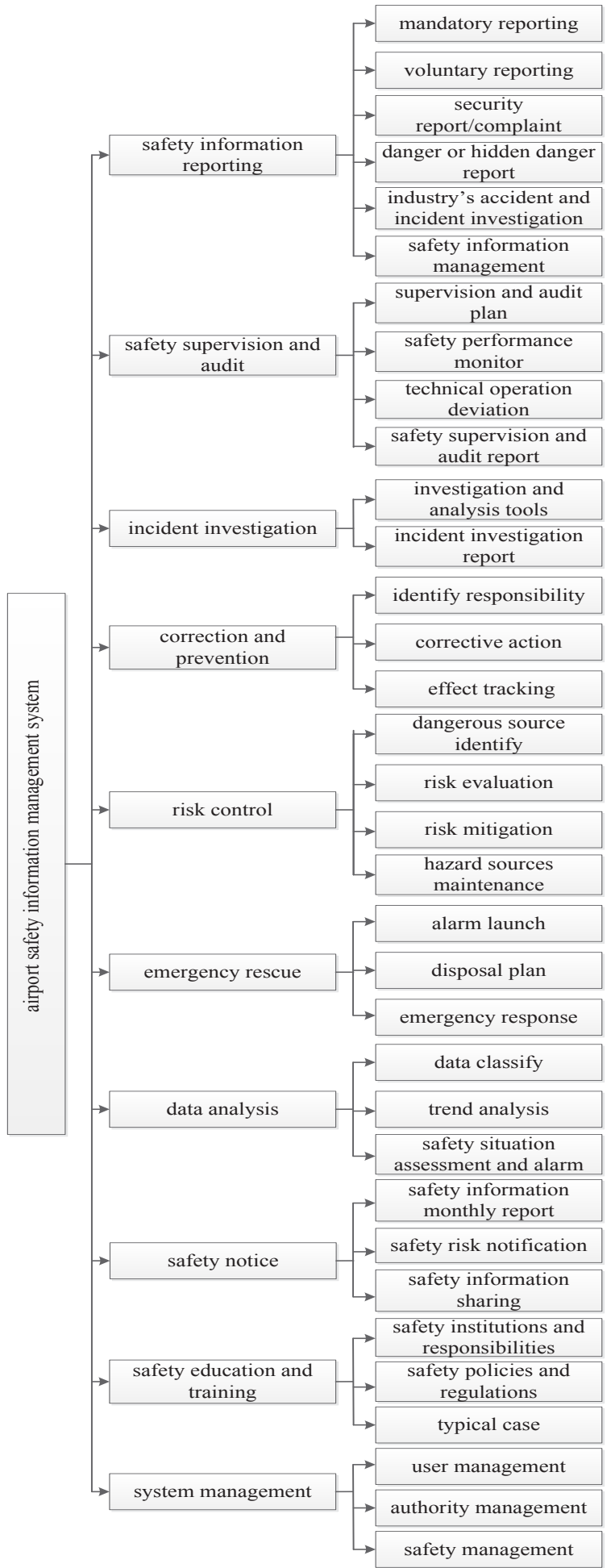

Fig. 3. Structure of functional modules of the system

\subsection{Incident investigation}

Incident investigation module will start, when unsafe events or risks are found through safety information analysis. Incident investigation module has functions of creating, editing, inquiring and automatically generating report. It can provide investigation and analysis tools to help safety experts to carry out event analysis and evaluation, and provide suggestions and measures. With approval by the leaders, it can trigger other safety management modules such as correction and prevention, risk control, emergency rescue, safety supervision and audit, safety education and training.

\subsection{Correction and prevention}

For the hidden danger which is classified as low hazard level by incident investigation module, corrective and preventive module can identify the responsibility, and issue the corrective notice to the relevant business departments. The relevant business departments should establish corrective actions and submit them to the module for verification. The module also has the function of track the completion of corrective actions.

\subsection{Risk control}

Risk control module carries out information maintenance of hazard sources. When the incident investigation module finds serious hazards, risk control module will launched to identify the dangerous source, using fuzzy clustering, artificial neural network, grey system and other methods to further assess the severity of the hazard, determine the risk level. The module can propose risk mitigation measures according to the evaluation and airport safety management requirements, and track the effectiveness of risk mitigation measures.

\subsection{Emergency rescue}

When a sudden and destructive emergency happens in the airport, emergency rescue module will star. The module alarms the relevant departments and staffs according to the event type. The module provides the disposal plan to help to make reasonable decisions on the rescue plan and resources. In the rescue process, with the module, the scene information can timely be fed back, so that the leader can grasp the progress of the rescue in real time. The module can also record the various decision-making and progress information in the emergency rescue process, so as to summarize the experience and lessons afterwards.

\subsection{Data statistics}

Data statistics module can classify the data according to the time interval, the severity of the incident, the event type and so on. It can also perform trend analysis. This can help decision-makers to find out the cause of event correlation, analyse safety trends, excavate safety laws, and obtain airport safety situation. When the airport reaches a certain safety threshold, the module can 
automatically alarm, and the relevant experts or leaders can judge and take countermeasures accordingly.

\subsection{Safety notice}

After certain treatment, the safety information can form the safety information monthly report and the safety risk notification, which is convenient for the communication of various departments of the airport. The module provides an open interface. Airport subsidiary can submit a safety information report to the group company. Information exchange can also be easily carried out between airport subsidiaries. Other business enterprises at the airport can also voluntarily join the airport information sharing.

\subsection{Safety education and training}

Safety education and training module includes airport safety institutions and responsibilities, safety operation policies and regulations, typical cases and so on. The module has full text browsing, keyword search and other functions, which can provide training materials for airport safety education and training, and contribute to the improvement of the overall safety level of the airport.

\subsection{System management}

System management module includes user management, authority management, safety management and other functions. Through it, the system administrator can set which users can use the system, which functions can be used. In the process of user's use, the module automatically records its operation track and forms a log.

\section{Supporting conditions for system operation}

Airport safety information management system can improve the level of airport safety management, but its effective operation also requires the airport to strengthen the following two aspects of construction.

(1) Enhance the ability of safety information personnel

With more and more safety information collected by airport safety information management system, the role of safety information personnel will become more and more important, and their ability requirements are also getting higher and higher. But the present situation is: safety information personnel lack of professional background or lack of training and experience of data mining. Data mining ability is weak, resulting that the analysis quality of information is not high, and it is difficult to make a convincing evaluation. Therefore, in order to effectively collect and apply safety information, the ability of safety information personnel should be enhanced.

(2) Build a positive safety information culture

Safety information culture is a culture that respects facts, praises rationality and emphasizes precision. Respecting data and respecting objective facts is the foundation of giving play to safety information. The airport should carry out the education on information report honesty, strengthen the consciousness of honest report, and improve the information report quality of staffs.

\section{Conclusions}

Airport safety information management system can realize the effective integration and maximum sharing of safety information, deepen the analysis and utilization of safety information, and provide decision support for airport safety. In this paper, the overall framework of airport safety information management system based on $\mathrm{B} / \mathrm{S}$ model is constructed, and the supporting conditions of system operation are proposed. However, the construction of the system still has a lot of work to do, the design of the system function needs to be further refined, the system module algorithm needs to be implemented. In addition, because airport has more than one computer management system, how to integrate the airport safety information management system and other systems needs further study.

\section{References}

1. Y. Q. Wang, analysis and design of unsafe event management system in civil airport, Journal of Safety Science and Technology, 6, (2010),163-166.

2. Y. Chen, research on airport-oriented aviation safety information system, Computer Applications and Software, 29, (2012),46-49.

3. Y. J. Shi, Y. Q. Chen, problems and countermeasures on aviation safety information management, Journal of Safety Science and Technology, 6, (2010),116-120.

4. M. Zhan, acquisition, integration and mining of aviation safety production data, Science and Technology for Development, 8, (2012),36-39.

5. R. Zhang, R. Cheng, Y. Fan, design and research of risk management system for air traffic management, Intelligent Computer and Applications, 6, (2016), 111-112.

6. X. Y. Qi, L. L. Liu, S. Y. Bao, on the evaluation of the safety information management and control capability with the civil aviation system, Journal of Safety and Environment, 2,(2017),565-572. 


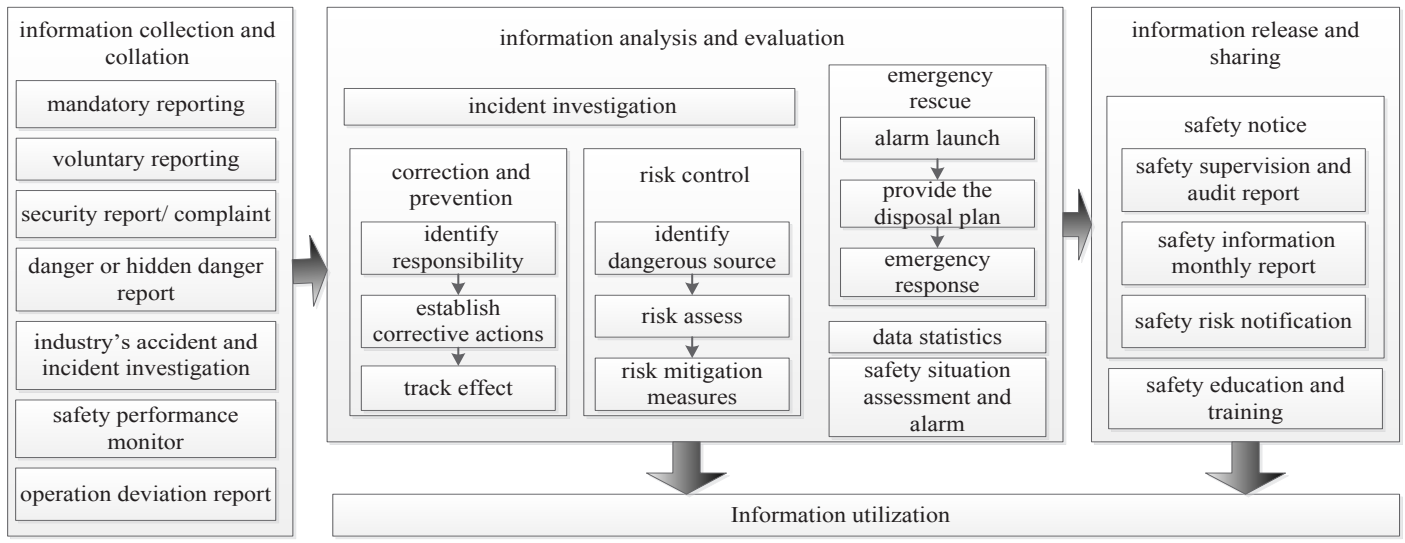

Fig. 2. Business process of the system 